\title{
Model for resonant photon creation in a cavity with time dependent conductivity
}

\author{
Martín Crocce, ${ }^{1}$ Diego A. R. Dalvit, ${ }^{2}$ Fernando C. Lombardo, ${ }^{3}$ and Francisco D. Mazzitelli ${ }^{3}$ \\ ${ }^{1}$ Department of Physics, New York University, \\ 4 Washington Place, New York, New York 10003 \\ ${ }^{2}$ Theoretical Division, MS B213 Los Alamos National Laboratory, Los Alamos, NM 87545 \\ ${ }^{3}$ Departamento de Física Juan José Giambiagi, \\ FCEyN UBA, Facultad de Ciencias Exactas y Naturales, \\ Ciudad Universitaria, Pabellón I, 1428 Buenos Aires, Argentina
}

(Dated: today)

\begin{abstract}
In an electromagnetic cavity, photons can be created from the vacuum state by changing the cavity's properties with time. Using a simple model based on a massless scalar field, we analyze resonant photon creation induced by the time-dependent conductivity of a thin semiconductor film contained in the cavity. This time dependence may be achieved by irradiating periodically the film with short laser pulses. This setup offers several experimental advantages over the case of moving mirrors.
\end{abstract}

PACS numbers: 03.70.+k;42.50.Lc;42.50.Nn

\section{INTRODUCTION}

In quantum field theory time-dependent boundary conditions or time-dependent background fields may induce particle creation, even when the initial state of a quantum field is the vacuum 1]. In the context of quantum electrodynamics, uncharged mirrors in accelerated motion can in principle create photons. This effect is referred to in the literature as the dynamical Casimir effect, or motion-induced radiation 2]. In particular, when the length of a high Q electromagnetic cavity oscillates with one of its resonant frequencies, the number of photons inside the cavity accumulates slowly and grows exponentially with time. Many authors have considered this problem using different approximations: from toy models of scalar fields in $1+1$ dimensions 3 to the more realistic case of scalar and electromagnetic fields in three dimensional cavities [4, 5]. Arbitrary periodic motion of the boundary of an ideal cavity has been studied in [6]. The relevance of finite temperature effects and losses have also been considered 7].

Unlike the static Casimir effect [8] , that has been measured with increasing precision in the last years $[9]$, an experimental verification of the dynamical counterpart is still lacking. The main reason is that typical resonance frequencies for microwave cavities are of the order of $\mathrm{GHz}$. It is of course very difficult to make a mirror to oscillate at such frequencies. Several alternative proposals have been investigated in which the physical properties of the medium inside the cavity change with time, but keeping fixed the boundary of the cavity. Yablonovitch [10 pointed out that a nonlinear optical medium whose index of refraction changes rapidly with time accelerates the vacuum fluctuations, and may create photons. Nonlinear optics may be used to produce effective fast moving mirrors.

Closer to our present work, Lozovik et al. 11] proposed that one could change effectively the length of a cavity by irradiating with ultra-short laser pulses a thin semiconductor film deposited on one of the walls of the cavity. They evaluated the number of photons created using an instantaneous approximation in which the cavity suddenly changes its length. Moreover, the conductivity of the thin film was assumed to vanish before excitation and to be infinite after irradiation. Cirone et al. [12] considered the photon generation produced by a time-dependent dielectric, using also an instantaneous approximation.

From an experimental point of view, the idea of changing the effective length of a cavity by irradiating a semiconductor is promising 13. The theoretical aspects of the problem are still not completely clear. The main goal of this paper is to analyze the proposal of Ref. 11] with a more realistic model that takes into account both the finite conductivity of the semiconductor film and the characteristic times of excitation 
and relaxation. We will also consider the possibility of periodic oscillations of the conductivity of the semiconductor, as this will enhance particle production for certain resonance frequencies.

We will therefore consider a rectangular cavity of fixed size, with a thin semiconductor film inside it. We will model the electromagnetic field by a massless scalar field and the film conductivity with a sharp potential. This model has been considered in previous works to describe partially transmitting mirrors [14], and it will be enough for our purposes. The modes for a massless scalar field inside such a cavity are described in Section II. When the film is irradiated with a short laser pulse its conductivity rapidly increases. After a relaxation time the conductivity drops down to the initial value. The irradiation-excitation-relaxation cycle can be repeated periodically. Although the conductivity may change several orders of magnitude, when the initial conductivity is high enough (as is the case for a semiconductor, see below), the time dependent frequency of the modes varies periodically with time with a small amplitude. This will allow us to use standard methods to compute the evolution of the modes inside the cavity, and to show that for resonant external frequencies the number of photons inside the cavity grows exponentially with time. This evolution is described in Section III. In Section IV we estimate the number of photons for realistic values of the different parameters, and we also discuss the experimental advantages of the proposal over the moving mirror case. Section $\mathrm{V}$ contains the conclusions of our work.

\section{THE MODEL}

We consider a three dimensional model of a massless scalar field within a rectangular cavity with perfect conducting walls with dimensions $L_{x}, L_{y}$, and $L_{z}$. At the mid point of the cavity $\left(x=L_{x} / 2\right)$ a thin film of semiconducting material is located. We model the conductivity properties of such material by a potential $V(t)$ : The ideal limit of perfect conductivity corresponds to $V \rightarrow \infty$, and $V \rightarrow 0$ to a 'transparent' material. In our more realistic scenario this potential varies between a minimum value, $V_{0}$, and a maximum $V_{\max }$. The Lagrangian of the scalar field within the cavity is given by

$$
\mathcal{L}=\frac{1}{2} \partial_{\mu} \phi \partial^{\mu} \phi-\frac{V(t)}{2} \delta\left(x-L_{x} / 2\right) \phi^{2},
$$

where $\delta(x)$ is the one-dimensional Dirac delta function. The use of an infinitely thin film is justified as long as the width of the slab is much smaller than the wavelengths of the relevant electromagnetic modes in the cavity. The corresponding Lagrange equation reads,

$$
\left(\nabla^{2}-\partial_{t}^{2}\right) \phi=V(t) \delta\left(x-L_{x} / 2\right) \phi .
$$

For the sake of clarity we divide the cavity into two regions: region I $\left(0 \leq x \leq L_{x} / 2\right)$ and region II $\left(L_{x} / 2 \leq x \leq L_{x}\right)$. Perfect conductivity at the edges of the cavity imposes the following boundary conditions

$$
\begin{aligned}
& \phi_{\mathrm{I}}(x=0, t)=\phi_{\mathrm{II}}\left(x=L_{x}, t\right)=0 \\
& \phi_{\mathrm{I}}(y=0, t)=\phi_{\mathrm{II}}\left(y=L_{y}, t\right)=0 \\
& \phi_{\mathrm{I}}(z=0, t)=\phi_{\mathrm{II}}\left(z=L_{z}, t\right)=0 .
\end{aligned}
$$

The presence of the semiconducting film introduces a discontinuity in the spatial derivative of the field (in the $\hat{x}$ direction), while the field itself remains continuous,

$$
\begin{aligned}
\phi_{\mathrm{I}}\left(x=L_{x} / 2, t\right) & =\phi_{\mathrm{II}}\left(x=L_{x} / 2, t\right), \\
\partial_{x} \phi_{\mathrm{I}}\left(x=L_{x} / 2, t\right)-\partial_{x} \phi_{\mathrm{II}}\left(x=L_{x} / 2, t\right) & =-V(t) \phi\left(x=L_{x} / 2, t\right) .
\end{aligned}
$$

This can be seen by integrating out the field equation (Eq. (21) in the neighborhood of the film. As we will see, there are two sets of solutions to Eqs. (34). One characterized by the functions

$$
\varphi_{\mathbf{m}}(\mathbf{x})=\sqrt{\frac{2}{L_{x}}} \sin \left(\frac{2 \pi m_{x} x}{L_{x}}\right) \sqrt{\frac{2}{L_{y}}} \sin \left(\frac{\pi m_{y} y}{L_{y}}\right) \sqrt{\frac{2}{L_{z}}} \sin \left(\frac{\pi m_{z} z}{L_{z}}\right),
$$


where $\mathbf{m}=\left(m_{x}, m_{y}, m_{z}\right)$ are positive integers, and the other by

$$
\psi_{\mathbf{m}}(\mathbf{x}, t)=\sqrt{\frac{2}{L_{x}}} \sin \left(k_{m_{x}}(t) x\right) \sqrt{\frac{2}{L_{y}}} \sin \left(\frac{\pi m_{y} y}{L_{y}}\right) \sqrt{\frac{2}{L_{z}}} \sin \left(\frac{\pi m_{z} z}{L_{z}}\right) .
$$

The function $\psi_{\mathbf{m}}$ depends on $t$ through $k_{m_{x}}(t)$, which is the $m_{x}$-th positive solution to the following transcendental equation

$$
2 k_{m_{x}} \tan ^{-1}\left(\frac{k_{m_{x}} L_{x}}{2}\right)=-V(t) .
$$

To simplify the notation, in what follows we will write $k_{m}$ instead of $k_{m_{x}}$.

Let us define

$$
\begin{gathered}
\Phi_{\mathbf{m}}(\mathbf{x})= \begin{cases}\varphi_{\mathbf{m}}(x, y, z) & 0 \leq x \leq L_{x} / 2 \\
\varphi_{\mathbf{m}}\left(x-L_{x}, y, z\right) & L_{x} / 2 \leq x \leq L_{x}\end{cases} \\
\Psi_{\mathbf{m}}(\mathbf{x}, t)= \begin{cases}\psi_{\mathbf{m}}(x, y, z, t) & 0 \leq x \leq L_{x} / 2 \\
-\psi_{\mathbf{m}}\left(x-L_{x}, y, z, t\right) & L_{x} / 2 \leq x \leq L_{x}\end{cases}
\end{gathered}
$$

These functions satisfy the boundary conditions Eq. (3) and Eq. (44), and the orthogonality relations $\left(\Phi_{\mathbf{m}}, \Phi_{\mathbf{n}}\right)=\delta_{\mathbf{m}, \mathbf{n}},\left(\Phi_{\mathbf{m}}, \Psi_{\mathbf{n}}\right)=0$, and $\left(\Psi_{\mathbf{m}}, \Psi_{\mathbf{n}}\right)=\left[1-\sin \left(k_{m}(t) L_{x}\right) / k_{m}(t) L_{x}\right] \delta_{\mathbf{m}, \mathbf{n}}$, where we have used the usual inner product in the intervals $\left[0, L_{i}\right]$. It is also easy to show that $\left(\Phi_{\mathbf{m}}, \frac{\partial \Psi_{\mathbf{n}}}{\partial k_{n}}\right)=\left(\Phi_{\mathbf{m}}, \frac{\partial^{2} \Psi_{\mathbf{n}}}{\partial k_{n}^{2}}\right)=0$.

For $t \leq 0$ the slab is not irradiated, consequently $V$ is independent of time and has the value $V_{0}$. The modes of the quantum scalar field that satisfy the Klein Gordon equation (2) are

$$
\begin{aligned}
v_{\mathbf{m}}(\mathbf{x}, t) & =\frac{e^{-i w_{\mathbf{m}} t}}{\sqrt{2 w_{\mathbf{m}}}} \Phi_{\mathbf{m}}(\mathbf{x}) \\
u_{\mathbf{m}}(\mathbf{x}, t) & =\frac{e^{-i \bar{\omega}_{\mathbf{m}} t}}{\sqrt{2 \bar{\omega}_{\mathbf{m}}}} \Psi_{\mathbf{m}}(\mathbf{x}, 0),
\end{aligned}
$$

where $w_{\mathbf{m}}^{2}=\pi^{2}\left[\left(\frac{2 m_{x}}{L_{x}}\right)^{2}+\left(\frac{m_{y}}{L_{y}}\right)^{2}+\left(\frac{m_{z}}{L_{z}}\right)^{2}\right], \bar{\omega}_{\mathbf{m}}^{2}=\left(k_{m}^{0}\right)^{2}+\left(\frac{\pi m_{y}}{L_{y}}\right)^{2}+\left(\frac{\pi m_{z}}{L_{z}}\right)^{2}$, and $k_{m}^{0}$ is the $m$-th solution to Eq. (17) for $V=V_{0}$. At $t=0$ the potential starts to change in time and the set $\left\{k_{m}\right\}$ of the eigenfrequencies of the cavity acquires a time dependence through Eq. (17).

Using Eqs. (10) and (11) we expand the field operator $\phi$ as

$$
\phi(\mathbf{x}, t)=\sum_{\mathbf{m}}\left[a_{\mathbf{m}} v_{\mathbf{m}}(\mathbf{x}, t)+b_{\mathbf{m}} u_{\mathbf{m}}(\mathbf{x}, t)+a_{\mathbf{m}}^{\dagger} v_{\mathbf{m}}^{*}(\mathbf{x}, t)+b_{\mathbf{m}}^{\dagger} u_{\mathbf{m}}^{*}(\mathbf{x}, t)\right],
$$

where $a_{\mathbf{m}}$ and $b_{\mathbf{m}}$ are annihilation operators. Notice that the solutions in Eq. (10) correspond to modes with a node at $x=L_{x} / 2$ and therefore their dynamics is not affected by the presence of the slab (if the initial particle number given by $\left\langle a_{\mathbf{m}}^{\dagger} a_{\mathbf{m}}\right\rangle$ is zero, it will remain zero for all times). These modes are also decoupled from the modes $u_{\mathrm{m}}$ thanks to the orthogonality conditions. Therefore, we will only consider the evolution of the modes given in Eq. (111).

For $t \geq 0$ we write the expansion of the field mode $u_{\mathrm{s}}$ as

$$
u_{\mathbf{s}}(\mathbf{x}, t>0)=\sum_{\mathbf{m}} P_{\mathbf{m}}^{(\mathbf{s})}(t) \Psi_{\mathbf{m}}(\mathbf{x}, t) .
$$


Replacing this expression into $\left(\nabla^{2}-\partial_{t}^{2}\right) u_{\mathbf{s}}=0$ we find

$$
\ddot{P}_{\mathbf{n}}^{(\mathbf{s})}+\omega_{\mathbf{n}}^{2}(t) P_{\mathbf{n}}^{(\mathbf{s})}=-\sum_{\mathbf{m}}\left[\left(2 \dot{P}_{\mathbf{m}}^{(\mathbf{s})} \dot{k}_{m}+P_{\mathbf{m}}^{(\mathbf{s})} \ddot{k}_{m}\right) g_{\mathbf{m n}}^{(A)}+P_{\mathbf{m}}^{(\mathbf{s})} \dot{k}_{m}^{2} g_{\mathbf{m n}}^{(B)}\right],
$$

where $\omega_{\mathbf{m}}^{2}(t)=k_{m}^{2}(t)+\left(\frac{m_{y} \pi}{L_{y}}\right)^{2}+\left(\frac{m_{z} \pi}{L_{z}}\right)^{2}$. Note that $\bar{\omega}_{\mathbf{m}}=\omega_{\mathbf{m}}(0)$. The coefficients $g_{\mathbf{m n}}^{(i)} \operatorname{read}$

$$
\begin{aligned}
& g_{\mathbf{m n}}^{(A)}=\frac{1}{\left(\Psi_{\mathbf{n}}, \Psi_{\mathbf{n}}\right)}\left(\frac{\partial \Psi_{\mathbf{m}}}{\partial k_{m}}, \Psi_{\mathbf{n}}\right), \\
& g_{\mathbf{m n}}^{(B)}=\frac{1}{\left(\Psi_{\mathbf{n}}, \Psi_{\mathbf{n}}\right)}\left(\frac{\partial^{2} \Psi_{\mathbf{m}}}{\partial k_{m}^{2}}, \Psi_{\mathbf{n}}\right) .
\end{aligned}
$$

Imposition of continuity of $u_{\mathbf{s}}$ and $\dot{u}_{\mathbf{s}}$ at $t=0$ gives the following initial conditions

$$
\begin{aligned}
& P_{\mathbf{m}}^{(\mathbf{s})}(0)=\frac{1}{\sqrt{2 \bar{\omega}_{\mathbf{m}}}} \delta_{\mathbf{s}, \mathbf{m}} \\
& \dot{P}_{\mathbf{m}}^{(\mathbf{s})}(0)=-i \sqrt{\frac{\bar{\omega}_{\mathbf{m}}}{2}} \delta_{\mathbf{s}, \mathbf{m}},
\end{aligned}
$$

provided that $V(t)$ and its time derivative are continuous at $t=0$.

\section{RESONANT PHOTON CREATION}

We are interested in the number of photons created inside the cavity. Hence we focus in resonance effects induced by periodic oscillations in the conductivity $V(t)$, which translates into effective periodic changes in the modes of the scalar field. Therefore we start by considering a time dependent conductivity given by

$$
V(t)=V_{0}+\left(V_{\max }-V_{0}\right) f(t)
$$

where $f(t)$ is a periodic and non negative function, $f(t)=f(t+T) \geq 0$, that vanishes at $t=0$ and attains its maximum at $f\left(\tau_{e}\right)=1$. In each period, $f(t)$ describes the excitation and relaxation of the semiconductor produced by the laser pulse. Typically, the characteristic time of excitation $\tau_{e}$ is the smallest time scale and satisfies $\tau_{e} \ll T$. We expand $f(t)$ in a Fourier series

$$
f(t)=f_{0}+\sum_{j=1}^{\infty}\left[l_{j} \cos (j \Omega t)+h_{j} \sin (j \Omega t)\right]=f_{0}+\sum_{j=1}^{\infty} f_{j} \cos \left(j \Omega t+c_{j}\right),
$$

where $\Omega=2 \pi / T$. Since $\tau_{e}$ is the smallest time scale, on general grounds we expect the first $T / \tau_{e}$ terms in the above series to be relevant. We will present a particular example in Section IV.

Under certain constraints, large changes in $V$ induce only small variations in $k$ through the transcendental relation between $k$ and $V$ (see Eq. (7)). In this case, a perturbative treatment is valid and a linearization of such relation is appropriate. Accordingly we write

$$
k_{n}(t)=k_{n}^{0}\left(1+\epsilon_{n} f(t)\right),
$$

where $\epsilon_{n}$ is obtained after replacing Eqs. (1719) into Eq. (7) and expanding it to first order in $\epsilon_{n}$. The result is

$$
\epsilon_{n}=\frac{V_{\max }-V_{0}}{L_{x}\left(k_{n}^{0}\right)^{2}+V_{0}\left(1+\frac{V_{0} L_{x}}{4}\right)} .
$$


The restriction for the validity of the perturbative treatment is $V_{0} L_{x} \gg V_{\max } / V_{0}>1$. These conditions are satisfied for realistic values of $L_{x}, V_{0}$, and $V_{\max }$ (see Section IV). It is worth noticing that we are interested in low eigenfrequencies, for which $k(t) \sim \mathcal{O}\left(L_{x}^{-1}\right)$. Nonetheless the perturbative treatment is also valid for $k \sim \mathcal{O}(V)$.

In what follows we will only consider expressions to first order in $\epsilon_{n}$. To analyze the possibility of parametric resonance we write the spectrum given in Eq. (19) as,

$$
k_{n}(t)=\tilde{k}_{n}^{0}\left(1+\epsilon_{n}\left(f-f_{0}\right)\right),
$$

where $\tilde{k}_{n}^{0} \equiv k_{n}^{0}\left(1+\epsilon_{\mathrm{n}} f_{0}\right)$ is a 'renormalized' frequency. The equation for the coefficients $P_{\mathbf{m}}^{(\mathbf{s})}(t)$ (Eq. (14) can now we written to first order in $\epsilon_{n}$ as,

$$
\ddot{P}_{\mathbf{n}}^{(\mathbf{s})}+\tilde{\omega}_{\mathbf{n}}^{2} P_{\mathbf{n}}^{(\mathbf{s})}=-2 \epsilon_{n}\left(k_{n}^{0}\right)^{2}\left(f-f_{0}\right) P_{\mathbf{n}}^{(\mathbf{s})}-\sum_{\mathbf{m}}\left[2 \dot{P}_{\mathbf{m}}^{(\mathbf{s})} \epsilon_{m} k_{m}^{0} \dot{f}+P_{\mathbf{m}}^{(\mathbf{s})} \epsilon_{m} k_{m}^{0} \ddot{f}\right] g_{\mathbf{m n}}^{(A)}+\mathcal{O}\left(\epsilon^{2}\right),
$$

where $\tilde{\omega}_{\mathbf{n}}^{2}=\left(\tilde{k}_{n}^{0}\right)^{2}+\left(\pi n_{y} / L_{y}\right)^{2}+\left(\pi n_{z} / L_{z}\right)^{2}$. This equation describes a set of coupled harmonic oscillators with periodic frequencies and couplings. It is of the same form as the equations that describe the modes of a scalar field in a three dimensional cavity with an oscillating boundary, and can be solved using multiple scale analysis (MSA) 4]. A naive perturbative solution of previous equations in powers of $\epsilon_{n}$ breaks down after a short amount of time (this happens for particular values of the external frequency such that there is a resonant coupling with eigenfrequencies of the cavity). In order to find a solution valid for longer times, we introduce a second time scale $\tau_{n}=\epsilon_{n} t$ and expand $P_{\mathbf{n}}^{(\mathbf{s})}$ to first order as $P_{\mathbf{n}}^{(\mathbf{s})}(t)=P_{\mathbf{n}}^{(\mathbf{s})(0)}\left(t, \tau_{n}\right)+\epsilon_{n} P_{\mathbf{n}}^{(\mathbf{s})(1)}\left(t, \tau_{n}\right)+\mathcal{O}\left(\epsilon_{n}^{2}\right)$.

The derivatives with respect to the time scale $t$ read

$$
\begin{gathered}
\dot{P}_{\mathbf{n}}^{(\mathbf{s})}=\partial_{t} P_{\mathbf{n}}^{(\mathbf{s})(0)}+\epsilon_{n}\left[\partial_{\tau_{n}} P_{\mathbf{n}}^{(\mathbf{s})(0)}+\partial_{t} P_{\mathbf{n}}^{(\mathbf{s})(1)}\right], \\
\ddot{P}_{\mathbf{n}}^{(\mathbf{s})}=\partial_{t}^{2} P_{\mathbf{n}}^{(\mathbf{s})(0)}+\epsilon_{n}\left[2 \partial_{\tau_{n} t}^{2} P_{\mathbf{n}}^{(\mathbf{s})(0)}+\partial_{t}^{2} P_{\mathbf{n}}^{(\mathbf{s})(1)}\right] .
\end{gathered}
$$

For the zeroth order term we get the equation of a harmonic oscillator

$$
P_{\mathbf{n}}^{(\mathbf{s})(0)}=A_{\mathbf{n}}^{(\mathbf{s})}\left(\tau_{n}\right) e^{i \tilde{\omega}_{\mathbf{n}} t}+B_{\mathbf{n}}^{(\mathbf{s})}\left(\tau_{n}\right) e^{-i \tilde{\omega}_{\mathbf{n}} t} .
$$

From Eq. (16) the initial conditions for $A_{\mathbf{n}}^{(\mathbf{s})}$ and $B_{\mathbf{n}}^{(\mathbf{s})}$ are

$$
\begin{aligned}
& A_{\mathbf{n}}^{(\mathbf{s})}\left(\tau_{n}=0\right)=\frac{1}{\sqrt{2 \bar{\omega}_{\mathbf{n}}}}\left(1-\frac{\bar{\omega}_{\mathbf{n}}}{\tilde{\omega}_{\mathbf{n}}}\right) \frac{\delta_{\mathbf{s}, \mathbf{n}}}{2} \approx \frac{1}{\sqrt{2 \bar{\omega}_{\mathbf{n}}}} \frac{\left(k_{n}^{0}\right)^{2}}{2 \bar{\omega}_{\mathbf{n}}^{2}} f_{0} \epsilon_{n} \delta_{\mathbf{s}, \mathbf{n}}, \\
& B_{\mathbf{n}}^{(\mathbf{s})}\left(\tau_{n}=0\right)=\frac{1}{\sqrt{2 \bar{\omega}_{\mathbf{n}}}}\left(1+\frac{\bar{\omega}_{\mathbf{n}}}{\tilde{\omega}_{\mathbf{n}}}\right) \frac{\delta_{\mathbf{s}, \mathbf{n}}}{2} \approx \frac{1}{\sqrt{2 \bar{\omega}_{\mathbf{n}}}}\left(1-\frac{\left(k_{n}^{0}\right)^{2}}{2 \bar{\omega}_{\mathbf{n}}^{2}} f_{0} \epsilon_{n}\right) \delta_{\mathbf{s}, \mathbf{n}} .
\end{aligned}
$$

The first order term $P_{\mathbf{n}}^{(\mathbf{s})(1)}$ satisfies

$$
\partial_{t}^{2} P_{\mathbf{n}}^{(\mathbf{s})(1)}+\tilde{\omega}_{\mathbf{n}}^{2} P_{\mathbf{n}}^{(\mathbf{s})(1)}=-2 \partial_{t \tau_{n}}^{2} P_{\mathbf{n}}^{(\mathbf{s})(0)}-2\left(k_{n}^{0}\right)^{2}\left(f-f_{0}\right) P_{\mathbf{n}}^{(\mathbf{s})(0)}-\sum_{\mathbf{m}} \frac{\epsilon_{m}}{\epsilon_{n}} g_{\mathbf{m n}}^{(A)}\left[2 \partial_{t} P_{\mathbf{m}}^{(\mathbf{s})(0)} k_{m}^{0} \dot{f}+P_{\mathbf{m}}^{(\mathbf{s})(0)} k_{m}^{0} \ddot{f}\right]
$$

where $g_{\mathbf{m n}}^{(A)}$ is calculated to zeroth order in $\epsilon$. The basic idea of MSA is to impose the condition that any term in the right-hand side of the previous equation with a time dependency of the form $e^{ \pm i \tilde{\omega}_{\mathbf{n}} t}$ must vanish. If not, these terms would be in resonance with the left-hand side term and secularities would appear. Applying this procedure we obtain

$$
\begin{aligned}
\frac{1}{2} \frac{d A_{\mathbf{n}}^{(\mathbf{s})}}{d \tau_{n}}= & \sum_{j} f_{j}\left\{-\frac{\left(k_{n}^{0}\right)^{2}}{4 i \tilde{\omega}_{\mathbf{n}}} B_{\mathbf{n}}^{(\mathbf{s})} e^{i c_{j}} \delta\left(2 \tilde{\omega}_{\mathbf{n}}-\Omega_{j}\right)-\sum_{\mathbf{m}} \frac{\epsilon_{m}}{\epsilon_{n}} \frac{\Omega_{j}}{4 i \tilde{\omega}_{\mathbf{n}}} g_{\mathbf{m} \mathbf{n}}^{(A)} k_{m}^{0}\left[\left(-\frac{\Omega_{j}}{2}-\tilde{\omega}_{\mathbf{m}}\right) A_{\mathbf{m}}^{(\mathbf{s})} e^{i c_{j}} \delta\left(\tilde{\omega}_{\mathbf{n}}-\tilde{\omega}_{\mathbf{m}}-\Omega_{j}\right)+\right.\right. \\
& \left.\left.\left(-\frac{\Omega_{j}}{2}+\tilde{\omega}_{\mathbf{m}}\right) A_{\mathbf{m}}^{(\mathbf{s})} e^{-i c_{j}} \delta\left(\tilde{\omega}_{\mathbf{n}}-\tilde{\omega}_{\mathbf{m}}+\Omega_{j}\right)+\left(-\frac{\Omega_{j}}{2}+\tilde{\omega}_{\mathbf{m}}\right) B_{\mathbf{m}}^{(\mathbf{s})} e^{i c_{j}} \delta\left(\tilde{\omega}_{\mathbf{n}}+\tilde{\omega}_{\mathbf{m}}-\Omega_{j}\right)\right]\right\}
\end{aligned}
$$


and

$$
\begin{aligned}
\frac{1}{2} \frac{d B_{\mathbf{n}}^{(\mathbf{s})}}{d \tau_{n}}= & \sum_{j} f_{j}\left\{\frac{\left(k_{n}^{0}\right)^{2}}{4 i \tilde{\omega}_{\mathbf{n}}} A_{\mathbf{n}}^{(\mathbf{s})} e^{-i c_{j}} \delta\left(2 \tilde{\omega}_{\mathbf{n}}-\Omega_{j}\right)+\sum_{\mathbf{m}} \frac{\epsilon_{m}}{\epsilon_{n}} \frac{\Omega_{j}}{4 i \tilde{\omega}_{\mathbf{n}}} g_{\mathbf{m n}}^{(A)} k_{m}^{0}\left[\left(-\frac{\Omega_{j}}{2}-\tilde{\omega}_{\mathbf{m}}\right) B_{\mathbf{m}}^{(\mathbf{s})} e^{-i c_{j}} \delta\left(\tilde{\omega}_{\mathbf{n}}-\tilde{\omega}_{\mathbf{m}}-\Omega_{j}\right)+\right.\right. \\
& \left.\left.\left(-\frac{\Omega_{j}}{2}+\tilde{\omega}_{\mathbf{m}}\right) B_{\mathbf{m}}^{(\mathbf{s})} e^{i c_{j}} \delta\left(\tilde{\omega}_{\mathbf{n}}-\tilde{\omega}_{\mathbf{m}}+\Omega_{j}\right)+\left(-\frac{\Omega_{j}}{2}+\tilde{\omega}_{\mathbf{m}}\right) A_{\mathbf{m}}^{(\mathbf{s})} e^{-i c_{j}} \delta\left(\tilde{\omega}_{\mathbf{n}}+\tilde{\omega}_{\mathbf{m}}-\Omega_{j}\right)\right]\right\} .
\end{aligned}
$$

The coupling coefficients as defined in Eq. (15) can be computed using Eqs. (617) and (19). However, we will not need the explicit expression in what follows.

Since the function $f(t)$ contains the frequencies $\Omega_{j}=j \Omega$, we expect resonant behavior and/or mode coupling when one of the following relations are satisfied

$$
\begin{aligned}
& \Omega_{j}=2 \tilde{\omega}_{\mathbf{n}} ; \\
& \Omega_{j}=\left|\tilde{\omega}_{\mathbf{n}} \pm \tilde{\omega}_{\mathbf{m}}\right|,
\end{aligned}
$$

for any pair of modes $\mathbf{m}$ and $\mathbf{n}$. As the eigenfrequencies $\tilde{\omega}_{\mathbf{n}}$ are not equidistant, if some of the conditions in Eq. (30) above are satisfied by a given set $(j, \mathbf{m}, \mathbf{n})$, in general they will not be satisfied for a different set $\left(j^{\prime}, \mathbf{m}^{\prime}, \mathbf{n}^{\prime}\right)$. This can be checked by inspection in each particular case. Assuming that this is the case, we can restrict the analysis to a single Fourier mode in the expansion [18), i.e. $f(t)=f_{0}+f_{j} \cos \left(j \Omega t+c_{j}\right)$.

Let us consider the most important case of parametric resonance, i.e. when the external frequency is tuned with some of the eigenfrequencies of the cavity, $\Omega_{j}=2 \tilde{\omega}_{\mathbf{n}}$. The contributions proportional to $g_{\mathbf{m}, \mathbf{n}}^{(A)}$ would be different from zero if there exists an integer $j^{\prime}$ such that $\left(2 \frac{j^{\prime}}{j} \pm 1\right) \tilde{\omega}_{\mathbf{n}}=\tilde{\omega}_{\mathbf{m}}$. This may happen only for some particular values of $L_{x}, L_{y}, L_{z}$. We will assume that the condition is not satisfied. Therefore, as mentioned above, we can restrict the analysis to the single $j$ Fourier mode. Moreover, the resonant mode $\mathbf{n}$ is not coupled and we are left with

$$
\frac{d A_{\mathbf{n}}^{(\mathbf{s})}}{d \tau_{n}}=i \frac{\left(k_{n}^{0}\right)^{2} f_{j} e^{i c_{j}}}{\Omega_{j}} B_{\mathbf{n}}^{(\mathbf{s})} ; \frac{d B_{\mathbf{n}}^{(\mathbf{s})}}{d \tau_{n}}=-i \frac{\left(k_{n}^{0}\right)^{2} f_{j} e^{-i c_{j}}}{\Omega_{j}} A_{\mathbf{n}}^{(\mathbf{s})} .
$$

The solution that satisfies the initial conditions Eqs. (26) is

$$
\begin{aligned}
A_{\mathbf{n}}^{(\mathbf{s})}\left(\tau_{n}\right) & =\frac{\delta_{\mathbf{s}, \mathbf{n}}}{\sqrt{8 \bar{\omega}_{\mathbf{n}}}}\left[\left(1-\frac{\bar{\omega}_{\mathbf{n}}}{\tilde{\omega}_{\mathbf{n}}}\right) \cosh \left(\frac{\left(k_{n}^{0}\right)^{2} f_{j}}{\Omega_{j}} \tau_{n}\right)+\left(1+\frac{\bar{\omega}_{\mathbf{n}}}{\tilde{\omega}_{\mathbf{n}}}\right) i e^{i c_{j}} \sinh \left(\frac{\left(k_{n}^{0}\right)^{2} f_{j}}{\Omega_{j}} \tau_{n}\right)\right] \\
& \approx \frac{i e^{i c_{j}} \delta_{\mathbf{s}, \mathbf{n}}}{\sqrt{2 \bar{\omega}_{\mathbf{n}}}} \sinh \left(\frac{\left(k_{n}^{0}\right)^{2} f_{j}}{\Omega_{j}} \tau_{n}\right) ; \\
B_{\mathbf{n}}^{(\mathbf{s})}\left(\tau_{n}\right) & =\frac{\delta_{\mathbf{s}, \mathbf{n}}}{\sqrt{8 \bar{\omega}_{n}}}\left[\left(1+\frac{\bar{\omega}_{\mathbf{n}}}{\tilde{\omega}_{\mathbf{n}}}\right) \cosh \left(\frac{\left(k_{n}^{0}\right)^{2} f_{j}}{\Omega_{j}} \tau_{n}\right)-\left(1-\frac{\bar{\omega}_{\mathbf{n}}}{\tilde{\omega}_{\mathbf{n}}}\right) i e^{-i c_{j}} \sinh \left(\frac{\left(k_{n}^{0}\right)^{2} f_{j}}{\Omega_{j}} \tau_{n}\right)\right] \\
& \approx \frac{\delta_{\mathbf{s} \mathbf{n}}}{\sqrt{2 \bar{\omega}_{\mathbf{n}}}} \cosh \left(\frac{\left(k_{n}^{0}\right)^{2} f_{j}}{\Omega_{j}} \tau_{n}\right) .
\end{aligned}
$$

The corresponding number of created photons with frequency $\tilde{\omega}_{\mathbf{n}}=\Omega_{j} / 2$ is given by

$$
\left\langle\mathcal{N}_{\mathbf{n}}(t)\right\rangle=\left\langle b_{\mathbf{n}}^{\dagger} b_{\mathbf{n}}\right\rangle=\sum_{\mathbf{s}} 2 \bar{\omega}_{\mathbf{n}}\left|A_{\mathbf{n}}^{(\mathbf{s})}(t)\right|^{2} \approx \sinh ^{2}\left(\frac{\left(k_{n}^{0}\right)^{2} f_{j}}{\Omega_{j}} \epsilon_{n} t\right),
$$

that leads to an exponential growth in the number of photons in the mode with frequency $\tilde{\omega}_{\mathbf{n}}$ at a rate $r_{\text {cond }}=2\left(k_{n}^{0}\right)^{2} f_{j} \epsilon_{n} / \Omega_{j}$. It can be shown that no other off-resonant frequencies $\Omega_{j^{\prime}} \neq \Omega_{j}$ present in the Fourier spectrum of $f(t)$ contribute to the exponential growth of the number of photons: in our model, the photon frequency spectrum $\mathcal{F}(\omega)$ develops an exponentially growing component at a single frequency $\omega=\Omega_{j} / 2$. 


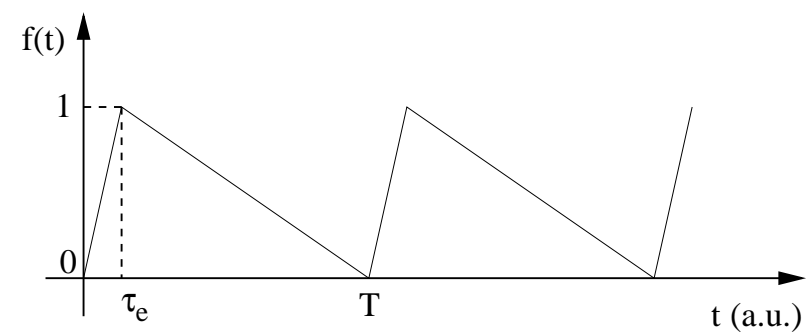

FIG. 1: Periodic excitation and relaxation of the semiconductor slab via ultra short laser pulses. The function $f(t)$ is proportional to the conductivity of the semiconductor (Eq. (17), and mimics its evolution in time.

\section{NUMERICAL ESTIMATIONS}

To determine the typical range of values of the conductivity $V$ before and after the short laser pulse is applied to the semiconductor we use the relation $V=4 \pi n_{s} e^{2} / m$, where $n_{s}$ is the surface charge density of free carriers in the semiconducting film, $e$ is the electron charge, and $m$ is the effective mass of the free carriers. This relation was derived in [14], where it was shown that our model Eq. (11) is equivalent to a planepolarized electromagnetic field propagating normally to an infinitesimally thin jellium-type plasma sheet. When a laser field suddenly impinges on the sheet, it produces time-dependent changes in the surface charge density $n_{s}(t)$ that induce a time variation in the conductivity $V(t)$ in our model. When the semiconductor is illuminated in the $\mathrm{GHz}$ range it becomes an excellent conductor (its conductivity being within $2 \%$ error from that of copper 15]). Therefore, using known values for the conductivities of good conductors, we can fix $V_{\max }=10^{16} \mathrm{~m}^{-1}$. When the laser field is not applied we can set $V_{0}=10^{10} \mathrm{~m}^{-1}-10^{13} \mathrm{~m}^{-1}$, the range of values for different semiconductors. For a cavity of size $L_{x} \simeq 10^{-2} \mathrm{~m}$, and when the ratio between the maximum and minimum conductivities is in the range $10^{3} \leq V_{\max } / V_{0} \leq 10^{6}$, we obtain from Eq. (20) the following range of values of $\epsilon, 10^{-8} \leq \epsilon \leq 10^{-2}$. Therefore, these small values give a posteriori justification for the perturbative approach we have used. Note that $\epsilon$ is small even if the conductivity of the film changes six orders of magnitude. For larger changes of the conductivity our perturbative approach is not adequate.

As we mentioned in the previous section, we consider a periodic function $f(t)$ with period $T$. To mimic the excitation and relaxation processes, we choose the following linear ramps

$$
f(t)=\left\{\begin{array}{cc}
\frac{t}{\tau_{e}} & \text { for } 0<t \leq \tau_{e} \\
\frac{(T-t)}{\left(T-\tau_{e}\right)} & \text { for } \tau_{e}<t \leq T
\end{array}\right.
$$

where $\tau_{e}$ is the excitation time scale, $\tau_{e} \ll T$. This simple choice will be enough to estimate the amplitude of the resonance effect. Expanding this $f(t)$ as in Eq. [18] we obtain $l_{j}=f_{j} \cos c_{j}=T\left[\cos \left(2 \pi j \tau_{e} / T\right)-\right.$ $1] /\left[2 \pi^{2} j^{2} \tau_{e}\left(1-\tau_{e} / T\right)\right]$, and $h_{j}=-f_{j} \sin c_{j}=T \sin \left(2 \pi j \tau_{e} / T\right) /\left[2 \pi^{2} j^{2} \tau_{e}\left(1-\tau_{e} / T\right)\right]$, so that

$$
f_{j}=\frac{1}{\pi j\left(1-\tau_{e} / T\right)}\left|\frac{\sin \left(\pi j \tau_{e} / T\right)}{\pi j \tau_{e} / T}\right| .
$$

On the one hand, in the limit $j \pi \tau_{e} / T \ll 1$ we get $f_{j} \approx 1 / \pi j$. This limit also corresponds to the instantaneous approximation $\tau_{e} \rightarrow 0$, in which the conductivity of the semiconductor slab suddenly changes from low to high values. As mentioned in the introduction, a similar instantaneous approximation was used in [11] and 12]; however, our approach is more general, not only because we consider finite conductivities of the slab, but also because we analyze periodic excitations via the laser pulses. On the other hand, for large values of $j$ the coefficients $f_{j}$ decay as $T / \tau_{e} j^{2} \pi^{2}$. We have computed the Fourier coefficients $f_{j}$ for the particular function $f(t)$ given in Eq. (34). However, provided that $1 \ll j \ll T / \pi \tau_{e}$, it can be shown that the result $f_{j} \sim 1 / \pi j$ is valid for any function $f(t)$ that grows on a time scale $\tau_{e}$ and relaxes on a time $T$. 
The number of created photons grows exponentially at a rate $r_{\text {cond }}=2\left(k_{n}^{0}\right)^{2} f_{j} \epsilon_{n} / \Omega_{j}$. When $L_{y}, L_{z} \gg L_{x}$ we have $k_{n}^{0} \approx \tilde{\omega}_{\mathbf{n}}=\Omega_{j} / 2$, so that the rate reads $r_{\text {cond }}=\Omega_{j} f_{j} \epsilon_{n} / 2$. In the limit $\Omega_{j} \tau_{e} \ll 1$ this rate is independent of $j$ and is given by $r_{\text {cond }}=\epsilon_{n} / T$. In the limit $\Omega_{j} \tau_{e} \gg 1$ it becomes $r_{\text {cond }}=2\left(\Omega_{j} \tau_{e}\right)^{-1} \epsilon_{n} / T$. In order to have resonant photon production for a cavity of $L_{x}=10^{-2} \mathrm{~m}$, the resonant frequency $2 \pi j / T$ must be in the $\mathrm{GHz}$ range. With the present technology, it is possible to have femtosecond laser pulses with a repetition frequency up to $100 \mathrm{MHz}$, thus the resonance could be achieved by rather low values of $j$, in the range $1-10$. The rate at which $\mathrm{GHz}$ photons would be produced depends now on whether the excitation time satisfies $\tau_{e} \ll 10^{-9} \mathrm{~s}$ or $\tau_{e} \gg 10^{-9} \mathrm{~s}$. Nowadays it is possible to reach values for $\tau_{e}$ as small as $10^{-12} \mathrm{~s}$ [15], thus yielding an estimated rate of $r_{\text {cond }} \sim 10^{8} \epsilon_{n} \mathrm{~Hz}$. Notice that this estimation remains true even for excitation times three orders of magnitude larger than $10^{-12} \mathrm{~s}$, after which there is a suppresion factor of order $1 / \Omega_{j} \tau_{e}$. Also notice that $r_{\text {cond }}$ is acceptably large in all the range where $\epsilon$ was assumed to vary, namely $10^{-8}-10^{-2}$.

This estimation for the rate $r_{\text {cond }}$ should be contrasted with that corresponding to the moving mirror case. In the latter case the typical rate is $r_{\mathrm{mov}} \approx \epsilon_{\mathrm{mov}} / T_{\mathrm{mov}}$, where $\epsilon_{\mathrm{mov}}$ is the relative amplitude of the oscillations of the mirror, and $T_{\text {mov }}$ is the period of oscillation of the moving mirror, which must be of order $10^{-9} \mathrm{~s}$ for a microwave cavity. If the oscillations are produced by deformations of the surface, the value of $\epsilon_{\text {mov }}$ cannot exceed $3 \times 10^{-8}[2]$. Then the accumulation of photons is a very slow process, which requires extremely high values for the $Q$-factor of the cavity. The ratio between the rate for photon creation for the time-dependent conductivity model and that of the moving mirror is

$$
\frac{r_{\mathrm{cond}}}{r_{\mathrm{mov}}} \approx \frac{\epsilon_{n}}{\epsilon_{\mathrm{mov}}} \frac{T_{\mathrm{mov}}}{T}
$$

which is of order $10^{6} T_{\mathrm{mov}} / T$ for $\epsilon_{n} \approx 10^{-2}$ and $\epsilon_{\mathrm{mov}} \approx 10^{-8}$. Even assuming that it is possible to produce oscillations of the mirror with a $\mathrm{GHz}$ frequency, and that the pulsing cycle of the laser is of some $\mathrm{MHz}$, this ratio is much larger than one.

Finally, in order to have resonance effects, the external frequency should be tuned with the frequency of the resonant mode with a high accuracy. In the moving mirror case, it has been shown $[4$ ] that if the external frequency is slightly detuned by an amount $\Delta \Omega_{\text {mov }}$ from the resonant frequency $\Omega_{\text {mov }}=2 \pi / T_{\text {mov }}$, then the number of photons grows exponentially as long as $\Delta \Omega_{\text {mov }} / \Omega_{\text {mov }}<\epsilon_{\text {mov }}$. A similar analysis shows that, in the setup described in this paper, an analogous restriction applies, with $\epsilon_{\text {mov }}$ replaced by $\epsilon_{n}$. As we have mentioned above, $\epsilon_{n}$ can take values much larger than $\epsilon_{\mathrm{mov}}$. Therefore, while the fine tuning is severe in the moving mirror case, it is not so critical for the case of a time dependent conductivity.

The discussion above shows that the present setup is much more promising for the experimental verification of the dynamical Casimir effect than the moving mirror one: the time dependent conductivity may be achieved with the present technology, the rate of photon creation is much larger, and the fine tuning is not so severe.

\section{CONCLUSIONS}

In this paper we have studied a simple scalar model to mimic photon creation induced by time-dependent changes in the conductivity of a thin semiconductor that is periodically excited by short laser pulses. When the conductivity of the semiconductor, placed inside a cavity of linear size in the cm range, changes up to six orders of magnitude during the excitation process induced by the laser pulses, the dynamical equations for the field modes have time dependent coefficients that oscillate in time with small amplitudes. This allowed us to solve the equations for the modes using multiple scale analysis, and to show that for some resonant excitation frequencies real photons are created inside the cavity, their number growing exponentially in time. Due to the very short excitation time of the semiconductor $\left(\tau_{e} / T \ll 1\right)$, it should be possible to tune a cavity mode with the frequency of a high $j$ Fourier harmonic of the time-dependent conductivity $V(t)$ of the semiconductor. Remarkably, as long as $j \pi \tau_{e} / T \ll 1$, the rate of photon creation is independent of $j$. It is then possible to produce resonant effects with ultra-short laser pulses whose pulsing frequency is well below 
the GHz range. We have also shown that this setup offers several advantages over the case of motion-induced radiation arising from moving mirrors, such as much faster photo-production rates and milder fine tuning problems.

Several issues remain to be investigated, such as how to extend this research to the more realistic full electromagnetic case, which involves Dirichlet and Neumann boundary conditions. Based on previous results for the moving mirror case [4], we expect an exponential growth of the number of created photons with different rates for transverse electric and transverse magnetic modes. Moreover, one should consider the macroscopic properties of the semiconductor slab, such as its frequency dependent conductivity and permittivity [16].

Another relevant aspect to investigate is how to disentangle Casimir photons from those that may be radiated from accelerated charges and thermal fluctuations of micro-currents in the semiconductor film, as this is being irradiated by the external laser pulse.

\section{ACKNOWLEDGMENTS}

F. C. L. and F. D. M. were supported by Universidad de Buenos Aires, CONICET, Fundación Antorchas

and Agencia Nacional de Promoción Científica y Tecnológica, Argentina. We would like to thank N. Bonadeo and G. Carugno for useful conversations.

[1] N. D. Birrell and P. C. D. Davies, Quantum Fields in Curved Space (Cambridge University Press, London, 1982).

[2] V. V. Dodonov, Adv. Chem. Phys. 119, 309 (2001).

[3] C. K. Law, Phys. Rev. Lett. 73, 1931 (1991); C. K. Law and W. C. Shieve, Phys. Rev. A 52, 4405 (1995); V. V. Dodonov, A. B. Klimov, and V. I. Man'ko, Phys. Lett. A 149, 225 (1990); A. Lambrecht, M.-T. Jaekel, and S. Reynaud, Phys. Rev. Lett. 77, 615 (1996); Europhys. Lett. 43, 147 (1998); D. A. R. Dalvit and F. D. Mazzitelli, Phys. Rev. A 57, 2113 (1998); 59, 3049 (1999); C.K. Cole and W.C. Schieve, Phys. Rev. A 64, 023813 (2001); M.A. Andreata, V.V. Dodonov, J. Phys. A 33, 3209 (2000); D.T. Alves, C. Farina and P.A.M. Neto, J. Phys. A 36, 11333 (2003).

[4] M. Crocce, D. A. R. Dalvit, and F. D. Mazzitelli, Phys. Rev. A 64, 013808 (2001); Phys. Rev. A 66, 033811 (2002)

[5] V. V. Dodonov and A. B. Klimov, Phys. Rev. A 53, 2664 (1996); D.F. Mundarain, P.A.M. Neto, Phys. Rev. A 57, 1379 (1998).

[6] A. V. Dodonov, E. V. Dodonov, and V. V. Dodonov, Phys. Lett. A 317, 378 (2003).

[7] G. Plunien, R. Schützhold, and G. Soff, Phys. Rev. Lett. 84, 1882 (2000); G. Schaller et al, Phys. Rev. A 66, 023812 (2002); R. Schützhold, G. Plunien and G. Soff, Phys. Rev. A 65, 043820 (2002).

[8] M. Bordag, U. Mohideen, and V. M. Mostepanenko, Phys. Rep. 353, 1 (2001).

[9] S. K. Lamoreaux, Phys. Rev. Lett. 78, 5 (1997); U. Mohideen and A. Roy, Phys. Rev. Lett. 81, 4549 (1998); H. B. Chan et al., Science 291, 1941 (2001); G. Bressi et al., Phys. Rev. Lett. 88, 041804 (2002); R. S. Decca et al., Phys. Rev. Lett. 91, 050402 (2003).

[10] E. Yablonovitch, Phys. Rev. Lett. 62, 1742 (1989).

[11] Y. E. Lozovik, V. G. Tsvetus, and E. A. Vinogradov, Physica Scripta 52, 184 (1995); JETP Lett. 61, 723 (1995).

[12] M.A. Cirone and K. Rzazewski, Phys. Rev. A 60, 886 (1999); M. Cirone, K. Rzazewski and J. Mostowski, Phys. Rev. A 55, 62 (1997).

[13] G. Carugno et al., MIR project, unpublished (2002).

[14] G. Barton and A. Calogeracos, Ann. Phys. (N.Y.) 238, 227 (1995).

[15] G. Carugno, private communication.

[16] M. Crocce, D. A. R. Dalvit, F.C. Lombardo, and F. D. Mazzitelli, in preparation. 